\title{
Automatización para el cálculo de indicadores: tasas de titulación y retención utilizados en la evaluación institucional estudio de caso Escuela Superior Politécnica de Chimborazo Riobamba - Ecuador \\ Automating the estimation of graduation and retention rates used for institutional evaluation. Case study at Escuela Superior Politécnica de Chimborazo Riobamba - Ecuador
}

\author{
Hernan Dario Centeno Aulla ${ }^{1 *}$, Juan Carlos Díaz Ordóñez $\mathbb{1}^{1}$, Gustavo Xavier Hidalgo \\ Solórzano ${ }^{1}$ \\ ${ }^{1}$ Dirección de Tecnologías de la Información y Comunicación, Escuela Superior Politécnica de \\ Chimborazo,Riobamba,Ecuador,EC060155; jcdiazo@espoch.edu.ec; gustavo.hidalgo@espoch.edu.ec
}

* Correspondencia: hernan.centeno@espoch.edu.ec

Recibido 14 abril 2019; Aceptado 27 mayo 2019; Publicado 06 junio 2019

Resumen:

Uno de los parámetros a cumplir por las Universidades y Politécnicas del Ecuador para su evaluación y acreditación son las tasas de titulación y retención, las mismas que se obtienen del número de estudiantes admitidos por primera vez en cada carrera, el número de estudiantes matriculados y el número de estudiantes titulados. Para el cálculo de estos indicadores es necesario el trabajo colaborativo de comisiones de carrera la misma que está compuesta por 5 o 6 docentes, es aquí donde nace la necesidad de hacer uso de las Tecnologías de la Información y la Comunicación (TIC) como una herramienta de gran utilidad en la automatización de procesos mediante la integración de sistemas informáticos, la Escuela Superior Politécnica de Chimborazo $(\mathrm{ESPOCH})$ en la actualidad dispone de 7 facultades, 38 carreras y cada carrera cuenta con un sistema académico de escritorio, siendo aquí donde se recopila la información necesaria para el cálculo de dichas tasas, en este sentido al sistema académico de escritorio se incluyó reportes que permiten el cálculo de estos indicadores, con el objetivo de optimizar el trabajo y garantizar la veracidad de la información obtenida en corto tiempo de respuesta. Por lo cual, como resultado se tiene la integración de reportes que muestran los indicadores de las tasas cumpliendo con las expectativas esperadas sobre la automatización de los reportes.

Palabras clave: Indicadores, reportes, retención, sistema informático, tasas de titulación.

Abstract:

Some of the parameters to be met by the Universities and Polytechnic Colleges in Ecuador throughout the evaluation and accreditation process are graduation and retention rates. These parameters derive from the number of students admitted for the first time in each major, the number of students enrolled and the number of students who exit the system. For the estimation of these parameters, it is necessary the collaborative work of career committees that are integrated by 5 or 6 faculty members. It is in this phase where the use of Information and Communication Technologies (ICT) as a tool is required as a tool that contributes to the automation of processes through the integration of computer systems. Chimborazo Polytechnic College (ESPOCH) currently has 7 colleges and 38 majors. Each major counts with its own academic desk system, where the necessary information for the estimation of the aforementioned rates is collected. In this sense, the academic desk system included reports that allow the estimation of these parameters. This study aimed to optimize the work and to guarantee the accuracy of the information obtained in a more efficient period. As a result, we have the integration of reports that show the parameters of the rates fulfilling the expectations related to the automation of the reports.

Keywords: Indicators, reports, retention rates, computer system, graduation rates 


\section{Unach}

\section{Introducción}

En la actualidad la educación superior presenta rasgos distintivos y experimenta desafíos muy semejantes (Días, 2007), en el caso de Ecuador desde la creación de la Constitución en el 2008 a la fecha, la educación ha presentado una serie de cambios significativos en su rol y su relación con la sociedad. Estos cambios han aportado para construir un nuevo marco de políticas públicas y un nuevo escenario en el cumplimiento y aplicación de conocimiento (Milia, 2014). Uno de los aspectos importantes a tomar en cuenta es la evaluación y acreditación institucional. La acreditación es una validación de vigencia quinquenal realizada por el Consejo de Aseguramiento de la Calidad de la Educación Superior (CACES), para certificar la calidad de las instituciones de educación superior, de una carrera o programa educativo, sobre la base de una evaluación previa (Asamblea Constituyente del Ecuador, 2010), por lo cual hoy en día la evaluación de la calidad institucional se constituye en uno de los grandes desafíos de la educación (Cabrera, 2005).

En este sentido, la concepción del modelo de evaluación, parte de una búsqueda del concepto de calidad en la educación superior en general y en particular de una definición de la calidad de la educación superior universitaria en el Ecuador (CACES, 2015). Considerando el criterio investigación, dentro del modelo de evaluación institucional, permite establecer el nivel que las universidades y escuelas politécnicas del Sistema de Educación Superior, han alcanzado en la generación de nuevo conocimiento a través de la investigación científica, lo cual constituye una de sus principales actividades (CACES, 2018)

Uno de los criterios de evaluación de las universidades del Ecuador es la eficiencia académica, que permite determinar las tasas de retención y eficiencia terminal que dichas Instituciones obtienen como resultado de las estrategias establecidas para sostener y acompañar a sus estudiantes durante todo el proceso educativo. Este criterio surge de la idea de que las Instituciones son responsables por el acompañamiento de sus estudiantes desde las instancias de nivelación hasta después de haber culminado la totalidad de sus créditos y estén listos para iniciar con sus trabajos de titulación (CACES, 2013).

Además, se debe considerar que la retención de estudiantes se ha convertido en uno de los temas de mayor interés investigativo en el ámbito de la educación superior, debido a las altas tasas de deserción que se están presentando desde hace varios años (Guevara, 2012). Este problema preocupa en todas las universidades, por ello muchas instituciones promueven estudios para analizar sus causas y desarrollan programas de retención de estudiantes, de los estudios analizados se deduce que el mayor porcentaje de abandono se da en el primer año de contacto con la universidad (Nuño et al., 2013).

El cálculo de indicadores de las tasas de titulación es un proceso que se debe llevar a cabo en todas las universidades del Ecuador para su evaluación, en este contexto se habla de la influencia del uso de las TIC como herramienta de apoyo para facilitar el trabajo de estos indicadores. Señalando que no hay medios mejores que otros si no que todos llegan a ser complementarios (Barroso et al., 2002), tomando en cuenta que, para adaptarse a las necesidades de la sociedad actual, las instituciones de educación superior deben flexibilizarse y desarrollar vías de integración de las tecnologías de la información y la comunicación en los procesos de formación (Salinas, 2004).

Considerando que, con el tiempo, las herramientas informáticas evolucionan con lo que surge la necesidad de actualizar los recursos para que se ajusten al entorno social. En donde se incluyen a las instituciones educativas, las cuales están en la obligación de ponerse a la vanguardia de la tecnología (Abarca et al., 2013), de esta manera, la rápida evolución de las TIC permite la aparición de nuevos productos y herramientas informáticas, utilizados en la gestión de procesos (Marco et al., 2002).

Por lo tanto, la implementación de un sistema académico en las instituciones tiene un gran impacto por ser un sistema académico encargado de gestionar todos los procesos que se realiza en la institución educativa, resultando ser una herramienta que ayudará a reducir el trabajo (Puma \& Vinicio, 2012). Se debe tomar en cuenta que un buen sistema de información ha de ser capaz de recibir y procesar los datos del modo más eficaz y sin errores (Trasobares, 2003).

Fundamentalmente un sistema debe ofrecer a sus usuarios la posibilidad de acceder a información necesaria para tomar decisiones, la capacidad de interactuar para efectuar transacciones generalmente basadas en información disponible (Tramullas, 2006), una manera factible de mostrar información a los usuarios de un sistema es en forma de reportes por la estructura que presenta ya que se componen de secciones, cada sección es un espacio horizontal en la página, cada reporte cuenta con diversos tipos de secciones (Sant'Anna, 2010). 


\section{Unach}

El objetivo de este estudio fue automatizar los indicadores para las tasas de titulación y retención utilizados en la evaluación institucional. Los objetivos específicos del estudio fueron: determinar la estructura del sistema de carrera que sirve para la gestión de notas de la ESPOCH, establecer herramientas para el desarrollo del sistema de carrera y servicios para la integración de reportes y mostrar reportes con las tasas de retención y titulación.

\section{Antecedentes}

Previo a la integración de reportes de los indicadores de las tasas de retención y titulación para la evaluación institucional en la Escuela Superior Politécnica de Chimborazo (ESPOCH) se describe a continuación cómo se realiza el cálculo de dichos indicadores. Para el cálculo de la tasa de retención de un primer año se entendió como un indicador frecuentemente utilizado a nivel internacional, dado que la mayor tasa de deserción se produce en este período. De manera simple, como un cociente entre el número de estudiantes que ingresan como alumnos de primer año a una carrera o programa en un año determinado, y el número de esos mismos estudiantes que se mantienen como estudiantes antiguos en la misma institución al año siguiente (CACES, 2016). Por otro lado, para la tasa de titulación, se conocen los procesos eficientes de docencia, investigación y vinculación permitiendo lograr eficiencia en la tasa de titulación de los estudiantes (CACES, 2015).

\section{Materiales y métodos}

\subsection{Método de investigación}

Para el desarrollo del presente trabajo se hizo uso del método inductivo, en este aspecto se observó que realizar el cálculo de estos indicadores requiere principalmente de la disponibilidad en los horarios de clases de los docentes que se dedican a realizar este proceso de forma manual, por lo tanto, con la automatización de dicho proceso se espera reducir este tiempo y obtener los valores exactos de las tasas.

\section{2 Área de estudio}

El estudio se llevó a cabo en la ESPOCH ubicada en el cantón Riobamba, perteneciente a la provincia de Chimborazo (figura 1). La ESPOCH es una institución con personería jurídica de derecho público totalmente autónoma, se rige por la Constitución Política del Estado Ecuatoriano, la Ley de Educación Superior y por su propio estatuto y reglamentos internos. Tiene por misión el formar profesionales e investigadores competentes, para contribuir al desarrollo sustentable del país.

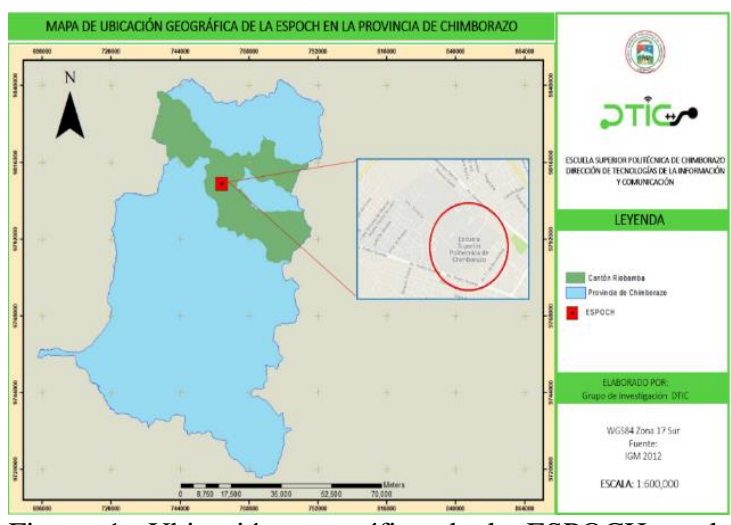

Figura 1: Ubicación geográfica de la ESPOCH en la provincia de Chimborazo.

La intención de la implementación de estos reportes es la automatización del cálculo de las tasas de retención y titulación que lleva a cabo en la ESPOCH durante el proceso de evaluación, para obtener estos indicadores se emplea las fórmulas (1) y (2) las mismas que se basan en el número de estudiantes admitidos en primer semestre, el número de estudiantes matriculados y el número de estudiantes titulados, para lograr con este objetivo en cada carrera de la ESPOCH se reunía comisiones de 5 o 6 docentes para determinar estos cálculos.

Ante esta situación y en vista que el sistema académico de gestión de notas guarda esta información por carreras se decidió integrar como un módulo más del sistema, de esta manera la información obtenida del sistema de notas sirve para alimentar a los reportes y generar la información deseada.

Por lo tanto para la integración de reportes de los indicadores en el sistema académico de carrera de la ESPOCH fue necesario analizar la estructura de dicho sistema determinando la siguiente estructura:

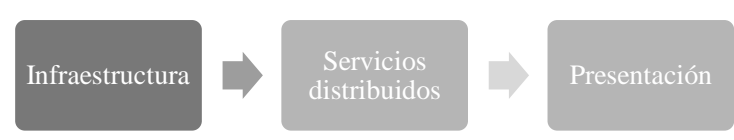

Figura. 2: Estructura del sistema de carrera de la ESPOCH.

La estructura del sistema de carrera de la ESPOCH como se puede observar en la figura 2 presenta un orden lógico, el mismo que está conformada por diferentes capas como la capa de infraestructura, en donde se definieron todas las entidades del sistema. En la capa de servicios distribuidos se tiene todos los servicios creados en base a la capa de infraestructura y en la capa de presentación se halla la interfaz de usuario que muestra toda la información del sistema, 
esta información es obtenida mediante los servicios definidos en la capa de servicios distribuidos, mismos que se encuentran publicados en el servidor de la institución. Siguiendo la lógica descrita se procedió a la creación e integración de los reportes de las tasas a través de la utilización de múltiples herramientas para el desarrollo del sistema académico, las cuales se presentan en la tabla 1.

Tabla 1: Herramientas para el desarrollo del sistema.

$\mathrm{N}^{\circ} \quad$ Herramientas

Características constructivas
Referencia

- Principal herramienta de trabajo para la administración y el desarrollo de aplicaciones.

Motor de base de - Es posible administrar de manera gráfica, aunque todas las tareas también se realizar utilizando scripts.

Gabillaud, 2015

datos SQL 2014 . Integra de manera nativa muchas herramientas que hacen que sea algo más que un simple servidor de base de datos relacionales.

- Es un IDE Entorno de desarrollo integrado para sistemas operativos

2 Visual Studio - $\begin{aligned} & \text { Windows. } \\ & \text { Soporta varios lenguajes de programación como lo son Visual C\# }\end{aligned}$ Visual J\#, C++, ASP.NET y Visual Basic .NET.

- Actualmente uno de los lenguajes de programación más populares.

3 Lenguaje de programación C\#

- Está disponible para el desarrollo de programas de propósito general, proporcionando al programador el nivel de abstracción preciso para abordar el desarrollo de cualquier aplicación por compleja.

Sierra, 2011

- Se basa en una plantilla, que consiste en un documento del tipo en que se quiere generar el reporte, y donde se define la estructura y el estilo de la información que se incluirá en el documento a partir de la fuente de datos.

Nobregas et al., 2013

- Es la versión más completa, que soporta hasta 64 procesadores.

- Se trata de una versión destinada a servidores potentes que están disponible bajo un programa de clave de licencia por volumen, está diseñado para ofrecer una plataforma flexible y completa.

Neild \& Van Jones, 2014

- Alternativa al diseño de servicios web, con mayor simpleza que SOAP y los servicios basados en WSDL, haciendo que las pruebas sobre esta arquitectura cobren mayor relevancia.

Mascheroni \& Irrazábal, 2016

- Herramienta que permite crear peticiones de manera muy sencilla, para luego poder enviarlas a un servidor y visualizar la respuesta.

- $\quad$ Es un cliente REST que puede integrarse con el navegador Google Chrome.
De esta manera, con las herramientas descritas se contribuyeron la capa de infraestructura creando una clase por reporte (tasa de titulación, tasa de retención e indicadores), dichas clases contiene toda la información de los reportes a desarrollar. En la capa de servicios distribuidos se creó una lista y un objeto de tipo clase que contiene la información a ser mostrada en el reporte, posteriormente se extrajo la información necesaria de la base de datos de carrera en formato JSON (JavaScript Object Notation) misma que viene en forma de vector.

Por lo cual, fue necesario recorrer con un bucle de repetición y asignar cada elemento del arreglo al objeto, luego se procedió a agregar a la lista de tipo clase al objeto, con esto se obtuvo una lista con la información deseada, finalmente en la capa de presentación se estableció el reporte esperado en el cual se puede observar los valores de las tasas de retención y titulación, además de los reportes el sistema muestra un listado de los estudiantes matriculados en las cohortes de evaluación.

\subsection{Cálculo de la tasa de retención}

Para el cálculo de la tasa de retención de la carrera que está siendo evaluada se escogieron los períodos de evaluación, mismos que estaban basados en los períodos académicos de la institución, lo cual sirvió para determinar la permanencia de un estudiante en la carrera que fue admitido por primera vez. 


\section{Unach}

La tasa indica que se debe recorrer cuatro semestres atrás sin tomar en cuenta el período de evaluación para identificar el inicio de la cohorte. En la tabla 2, se muestra un cómo se seleccionaron los períodos de evaluación y de la cohorte de inicio.

Tabla 2: Períodos de académicos analizados de la ESPOCH.

\begin{tabular}{lll}
$\mathrm{N}^{\circ}$ & \multicolumn{1}{c}{ Semestres } & \multicolumn{1}{c}{ Criterio } \\
\hline 1 & Abril 2017 - Agosto 2017 & Evaluación \\
2 & Octubre 2016 - Marzo 2017 & \\
3 & Abril 2016 - Agosto 2016 & \\
4 & Octubre 2015 - Marzo 2016 & \\
5 & Abril 2015 - agosto 2015 & Cohorte \\
6 & Octubre 2014 - Marzo 2015 & \\
\hline
\end{tabular}

Una vez identificada la cohorte se obtuvo:

- NTEA. Número de estudiantes que ingresaron a primer semestre en el periodo de la cohorte.

- NEMA. Número de estudiantes matriculados en el periodo de evaluación.

Finalmente se aplicó la fórmula para obtener la tasa de retención:

$$
T R=\frac{N E M A}{\text { NTEA }} * 100
$$

Donde el valor de TR indican el porcentaje de estudiantes que permanecen en la carrera después de dos años de haber sido admitidos.

\subsection{Cálculo de la tasa de titulación}

Para el cálculo de esta tasa, de igual forma se escogió el periodo de evaluación, para determinar la cohorte se debe considerar el número de semestres de duración de cada carrera, a este valor se adiciona 3 semestres que establece el reglamento de educación superior del Ecuador para que se titule un estudiante, de esta manera se obtiene el número se semestres a recorrer obteniendo los siguientes datos:

- NEC. Número de estudiantes que ingresaron a primer semestre en el periodo de la cohorte establecido ( $\mathrm{n}$ semestres antes del periodo de evaluación escogido).

- NEG. Número de estudiantes que ingresaron en la cohorte y se graduaron antes de la fecha fin del periodo de evaluación.

Finalmente se aplicó la siguiente fórmula para hacer el cálculo de la tasa de titulación:

$$
T G=\frac{N E G}{\mathrm{NEC}} * 100
$$

En donde el valor de TG indica el porcentaje de estudiantes que se titularon dentro del rango establecidos.

Es importante manifestar que anteriormente este proceso se llevaba a cabo en forma manual; es decir los directores de las 38 carreras de la institución designaban una comisión conformada por algunos docentes quienes eran los responsables de determinar los indicadores. Para lo cual debían tomar la información de cada uno de los estudiantes contenida en las carpetas físicas personales que se encuentran en las secretarias de cada carrera; obteniendo datos del estudiante como: el semestre en el que ingresó por primera vez a la institución, el semestre en el que se encuentra actualmente matriculado, el año de graduación, entre otros.

Posteriormente al conseguir dicha información se procedía a realizar el cálculo de las tasas y consecuentemente a la presentación de un informe con los indicadores generados para su respectivo análisis en la Dirección de Evaluación Académica. Por ende, el trabajo descrito anteriormente posee desventajas como: mucho tiempo invertido, varios docentes involucrados en comisiones y a su vez si la información calculada resultaba errónea al encontrar el error era tedioso y extenso.

En función a los antecedentes aludidos se realizó la petición al director de la Dirección de Tecnologías de la Información y Comunicación (DTIC) manifestando que se incluyan reportes con los indicadores de las tasas de retención y titulación en el sistema académico de secretaría de carrera. En correspondencia a la solicitud recibida, el director designó al equipo de profesionales que conforman el área de desarrollo para realizar el trabajo.

De esta manera como resultado se tuvo la implementación de reportes de manera satisfactoria, para lo cual se hizo uso de las bases de datos de carrera, mismas que almacenan la información de toda la vida estudiantil de cada alumno para lo cual se filtraron los datos de varias tablas para ser mostrados en los reportes solicitados.

\section{Servicios usados en la creación de reportes}

Para el desarrollo de los servicios a ser utilizados en la integración de reportes de indicadores se hizo uso 


\section{Unach}

de servicios REST, los mismos que son expresados en formato JSON debido que la serialización y desacralización de objetos es un mecanismo por el cual un objeto pasa de ser objeto a texto y puede ser transportado a cualquier destino, finalmente se vuelve a transformar a objeto, dicha información teórica resulta importante, ya que enriquecería más el tema para los usuarios de JSON en el desarrollo de sus aplicaciones (Castillo, 2016).

Para llevar a cabo la integración de estos servicios JSON se hizo uso de Entity Framework de Microsoft que es una plataforma para programar datos que eleva el nivel de abstracción del nivel relacional al nivel conceptual (entidad), por lo tanto reduce significativamente la falta de coincidencia de impedancia para aplicaciones y servicios centrados en datos (Adya et al., 2007). Los servicios después de ser creados fueron probados a través de los servicios Postman que muestra cómo sería el servicio en producción; verificando de esta manera que la información proporcionada es correcta.

Para la generación de los reportes se desarrollaron varios servicios detallados en la tabla 3 .

Tabla 3: Servicios para la generación de reportes.

\begin{tabular}{|c|c|c|}
\hline $\mathrm{N}^{\circ}$ & Servicios & Información proporcionada \\
\hline 1 & Dato estudiante & $\begin{array}{l}\text { - Información personal del estudiante como sus nombres, fecha de nacimiento, } \\
\text { identificación y otros. }\end{array}$ \\
\hline 2 & $\begin{array}{l}\text { Lista estudiantes con } \\
\text { primera matrícula }\end{array}$ & $\begin{array}{l}\text { - Una lista de estudiantes matriculados por primera vez en carrera dado un } \\
\text { período establecido por el usuario del sistema. }\end{array}$ \\
\hline 3 & Ver matrícula estudiante & $\begin{array}{l}\text { - Muestra el semestre más bajo que se encuentra matriculado un estudiante dado } \\
\text { un período establecido por el usuario del sistema. }\end{array}$ \\
\hline 4 & $\begin{array}{l}\text { Buscar estudiante } \\
\text { convalidación }\end{array}$ & $\begin{array}{l}\text { - Si un estudiante tiene convalidaciones en el periodo de evaluación en caso de } \\
\text { tener convalidaciones el estudiante es descartado de las tasas. }\end{array}$ \\
\hline
\end{tabular}

\section{$5 \quad$ Resultados}

Los servicios se desarrollaron bajo el Entorno de desarrollo integrado de desarrollo (IDE) de Visual Studio para lo cual se creó un nuevo servicio para alojar todos los métodos necesarios entre los que se encuentran: Lista de estudiantes de referencia, datos de los estudiantes y lista de estudiantes con primera matricula. Con el fin de obtener la información requerida, al crear un nuevo servicio en Visual Studio se crea la interfaz y la implementación (figura $3)$.

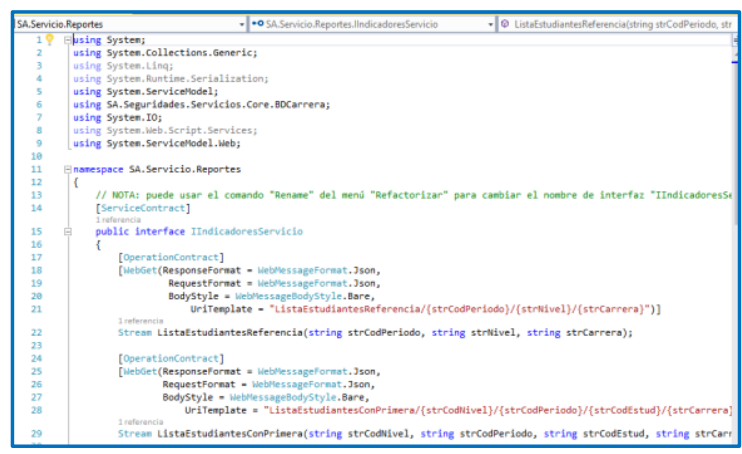

Figura. 3: Interfaz de los servicios.

De igual manera la implementación de los servicios corresponde a la interfaz en esta parte se hace las consultas a la base de datos y la información obtenida se expone en formato JSON (figura 4).

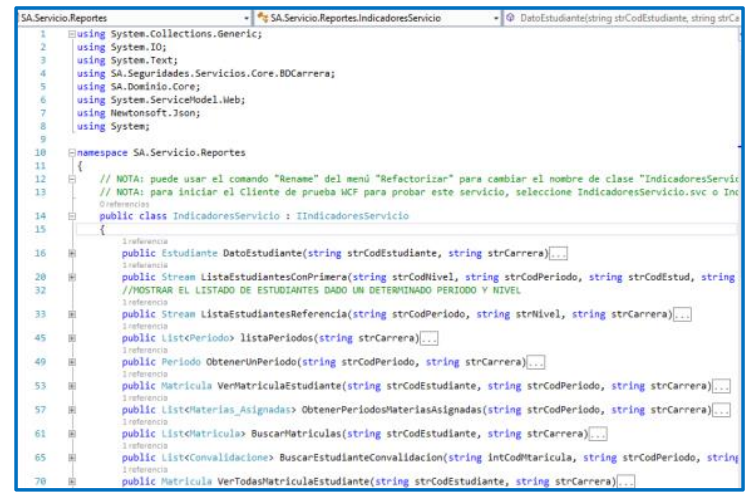

Figura. 4: Implementación de los servicios.

Los servicios implementados se encuentran alojados en los servidores de la ESPOCH para su respectivo consumo desde cada sistema de carrera de la institución.

\subsection{Implementación}

Los reportes generados fueron integrados al sistema académico de cada carrera de la institución, manifestando que en el menú de reportes se agregó un submenú de indicadores el cual brinda dos opciones que son: tasa de retención y titulación (figura 5). 


\section{Unach}

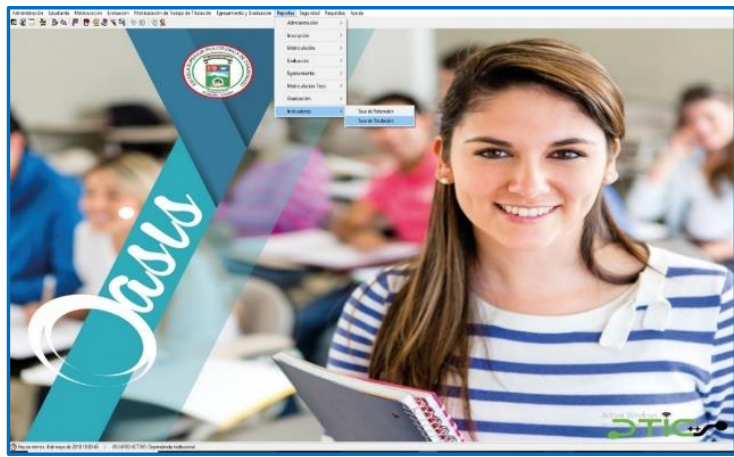

Figura. 5: Pantalla principal del sistema de carrera.

Una vez seleccionado el tipo de reporte deseado por el usuario, el siguiente paso es elegir el periodo o periodos a ser evaluados, de esta forma el sistema realiza el cálculo respectivo (figura 6). Una de las ventajas de hacer uso de estos reportes es que no solo indica el indicador de las tasas (figura 7), sino que también muestra el listado de los estudiantes admitidos en los periodos de las cohortes y el listado de estudiantes matriculados o titulados en los periodos de evaluación (figura 8).

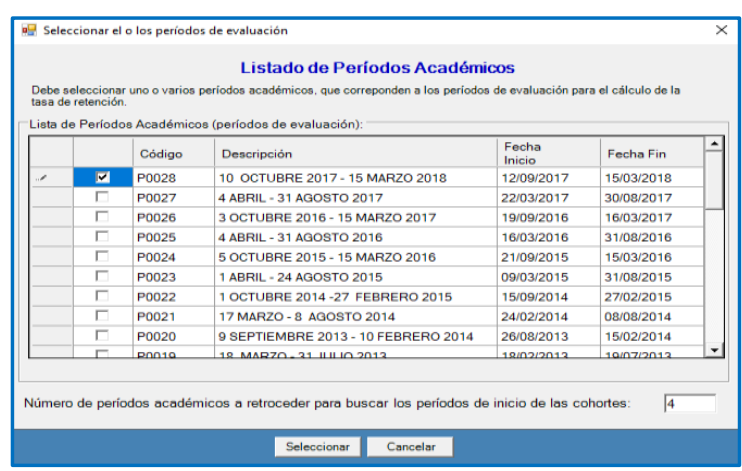

Figura. 6: Periodos académicos de la ESPOCH.

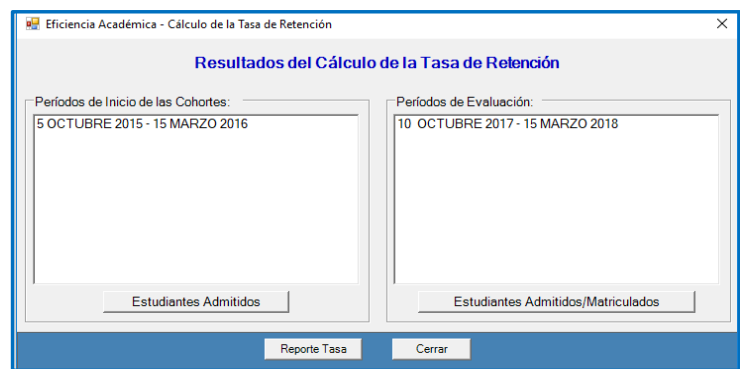

Figura. 7: Reportes a ser mostrados por el sistema.

Un aspecto en particular es que se puede visualizar un reporte con una lista de estudiantes admitidos en primer nivel en un determinado periodo.

A raíz del proceso expuesto, el resultado final es un reporte con los indicadores de la tasa de retención, que a su vez el valor obtenido es representado en porcentaje (figura 9).

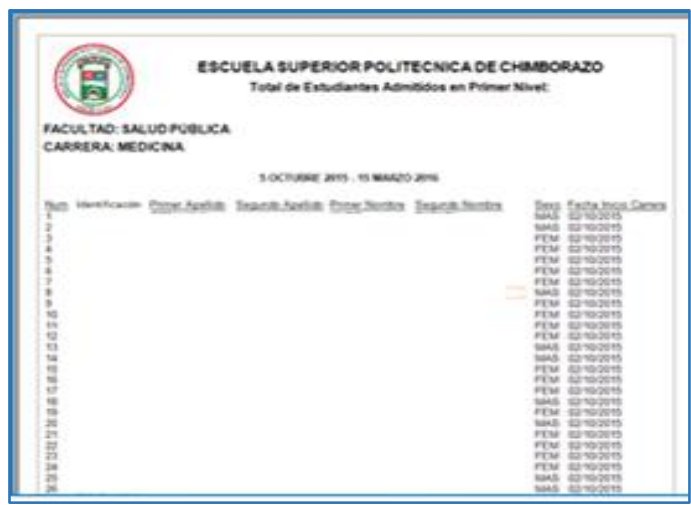

Figura. 8: Reporte de estudiantes admitidos. Con la finalidad de conservar la confidencialidad de la información se ha ocultado la identidad de los estudiantes

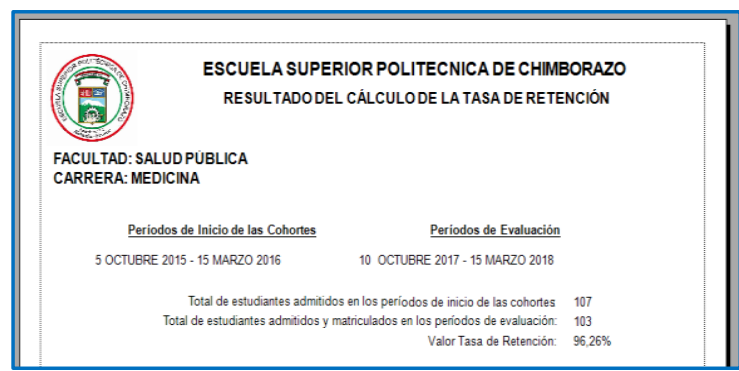

Figura. 9: Indicadores de la tasa de retención generados.

Las primeras pruebas fueron realizadas en la carrera de Medicina en donde el desarrollador responsable de realizar los reportes de los indicadores se reunió con la comisión de carrera. En este ambiente de trabajo los docentes de la comisión de carrera tenían a su disposición las actas físicas de los estudiantes matriculados en el período de evaluación y las actas de los estudiantes matriculados en primer semestre el período de la cohorte.

El proceso consta en identificar el número de estudiantes admitidos por primera en el período de cohorte, el siguiente paso determinar es número de estudiantes admitidos por primera vez y que se encuentran matriculados en el período de evaluación, una vez obtenidos estos valores se procedió al cálculo de la tasa de retención, todo este proceso demanda un tiempo estimado de dos a tres horas de trabajo en una comisión de carrera compuesta de 5 o 6 docentes.

Con la implementación de los reportes el programador se demoró un tiempo estimado de 5 segundos en obtener los valores de las tasas, los resultados obtenidos en el sistema fueron iguales a los resultados que calculaban los docentes en forma manual en comisiones de carrera, de esta manera se validó que los resultados arrojados en los reportes son los esperados los mismos que se obtuvieron en 


\section{Unach}

menor tiempo y sin la necesidad del trabajo colaborativo de una comisión de carrera.

\section{Acciones que el usuario puede realizar con el} sistema:

- Seleccionar uno o varios periodos de evaluación.

- Visualizar un reporte con la lista de estudiantes admitidos por primera vez en la ESPOCH.

- Observar un reporte con la lista de estudiantes que permanecen en la carrera que ingresaron por primera vez.

- Visualizar un reporte con la lista de estudiantes que ingresaron a la carrera y se titularon en la carrera de inicio de estudio en el tiempo establecido por las normas de educación superior.

- Apreciar un reporte con el número de estudiantes admitidos y estudiantes que permanecen en la carrera y su equivalente en porcentaje a la permanencia en la carrera.

- Generar un reporte con el número de estudiantes admitidos y estudiantes que se titularon en la carrera de inicio de estudios y su equivalente en porcentaje al número de titulados.

\section{Conclusiones}

En base a los objetivos específicos del estudio se llega a las siguientes conclusiones:

Se automatizó el proceso del cálculo de las tasas de retención y titulación por medio de reportes, cuya información es necesaria para el proceso de evaluación institucional y de carrera. Para la implementación del cálculo de estos indicadores se creó un módulo en el sistema académico de carrera de la ESPOCH, esto se debe que este sistema dispone de la información necesaria que permite determinar estos indicadores, por lo tanto, dichos reportes estarán disponibles en todas las secretarías de carrera de la ESPOCH reduciendo el tiempo de la obtención de estos valores.

Se identificó y analizó la estructura del sistema de carrera, dicha estructura consta de una capa de infraestructura, servicios distribuidos y la capa de presentación, por lo tanto, se tuvo que acoplar a esta estructura la integración de los reportes de las tasas de retención y titulación.

En cuanto a la capa de servicios distribuidos se crearon las consultas a la base de datos SQL devolviendo como respuesta los servicios, los cuales son consumidos desde la capa de infraestructura que sirve como capa intermedia siendo aquí donde se define el diseño de los reportes para que sean visualizados en la capa de presentación.

Para el desarrollo e integración de los reportes de tasa de retención y titulación se identificó como herramientas: el IDE de desarrollo de Visual Studio y el motor de base datos SQL, dichas herramientas son usadas en la Dirección de Tecnologías de la Información y Comunicación de la ESPOCH.

\section{Conflicto de Intereses}

Se expresa de manera libre y voluntaria que en el desarrollo del presente artículo no existen conflictos de interés personales por parte de ninguno de los autores por lo que se autoriza la publicación del trabajo.

\section{Agradecimientos}

Los investigadores agradecen de manera encarecida a la Dirección de Tecnologías de la Información y Comunicación de la Escuela Superior Politécnica de Chimborazo, por la apertura y el apoyo brindado durante el desarrollo del sistema generado.

\section{Referencias}

Abarca, E., Huezo, D. \& Mejía, J. (2013). Sistema informático para el control administrativo del complejo educativo "Federico González" en el municipio de San Sebastián departamento de San Vicente (Tesis doctoral). Facultad Multidisciplinaria Paracentral de la Universidad de El Salvador, San Vicente, El Salvador.

Adya, A., Blakeley, J., Melnik, S. \& Muralidhar, S. (2007). Anatomy of the ado. net entity framework. In Proceedings of the 2007 ACM SIGMOD international conference on Management of data. pp. 877-888. ACM

Asamblea Constituyente del Ecuador (2010). Registro oficial (Organo del Gobierno del Ecuador), año IIQuito, jueves 14 de Septiembre del 2006-no 356; Ley organica reformatoria a la ley orgánica de defensa del consumidor.

Barroso, J., Cabero, J. \& Romero, R. (2002). Las personas mayores y las nuevas tecnologías: una acción en la sociedad de la información. Revista Innovación Educativa, XXII, 319- 337.

Cabrera, V. (2005). El concepto calidad en la educación universitaria: clave para el logro de la competitividad institucional. Revista Iberoamericana de Educación, 36(12), 1-7.

Castillo, J. (2016). Serialización/desacralización de objetos y transmisión de datos con JSON: una 


\section{Unach}

revisión de la literatura. Tecnología en Marcha, 29(1), 118-125.

CACES. Consejo de Aseguramiento de la Calidad de la Educación Superior. (2015). Acreditación y Aseguramiento de la Calidad de la Educación Superior. Modelo de Evaluación Institucional de Universidades y Escuelas Politécnicas.

CACES. Consejo de Aseguramiento de la Calidad de la Educación Superior. (2015). CACES adoptó cinco criterios de evaluación a las universidades. EcuadorUniversitario.Com [Online]. Recuperado de: http://ecuadoruniversitario.com/deinstituciones-del-estado/ceaaces/ceaaces-adoptocinco-criterios-de-evaluacion-a-lasuniversidades/. [Acceso: 05-Marzo-2019].

CACES. Consejo de Aseguramiento de la Calidad de la Educación Superior. (2013). Informe General sobre la Evaluación, Acreditación, y Categorización de las Universidades y Escuelas Politécnicas.

CACES. Consejo de Aseguramiento de la Calidad de la Educación Superior (2011). Nueva fórmula de distribución de recursos a favor de las Universidades y Escuelas Politécnicas.

CACES. Consejo de Aseguramiento de la Calidad de la Educación Superior. (2015). Proceso de evaluación, recategorización, acreditación y categorización. p. 66.

Dias, J. (2007). Acreditación de la educación superior en América Latina y el Caribe. Madrid-BarcelonaMéxico: GUNI, Mundi-Prensa.

Gabillaud, J. (2015). SQL Server 2014: Administración de una base de datos transaccional con SQL Server Management Studio. Barcelona: Ediciones ENI.

Guevara, L. (2012). Retención estudiantil en la educación superior: revisión de la literatura y elementos de un modelo para el contexto colombiano. Colombia: Editorial Pontificia Universidad Javeriana.

Marco, F., Lacruz, M. \& Cortés, A. (2002). Representación y organización del conocimiento. La revolución digital en las bibliotecas y los centros de documentación. Universidad de Zaragoza. Facultad de Filosofía y Letras.

Mascheroni, M. \& Irrazábal, E. (2016). Framework para la creación y ejecución de pruebas automatizadas sobre servicios REST. In XXII Congreso Argentino de Ciencias de la Computación (CACIC 2016).

Milia, M. (2014). Marco de Políticas Públicas de Ciencia, Tecnología y Educación Superior en el Ecuador. Nuevos horizontes: dinámicas y condicionamientos para una Investigación Universitaria de cara a la Sociedad. Congreso Iberoamericano de Ciencia, Tecnología, Innovación y Educación, Buenos Aires. Ponencia.
Neild, T. \& Van Jones, M. (2014). Windows Server 2012 R2: Administración avanzada. Ediciones ENI.

Nobregas, K., Díaz, R. \& Mora, M. (2013). Sharereport: framework extensible orientado a la generación de reportes y la recuperación de información desde documentos. Editions thinking the future. Convencion y feria internacional de Ciencias Computacionales e Informáticas.

Nuño, A., García, A., Blanco, J., Casaravilla, A., Castejon, Á., Mahillo, Á. \& Malinga, M. (2013). Protocolo de calidad para la tasa de permanencia a un año en la Universidad Politécnica de Madrid. III Conferencia Latinoamericana sobre el abandono en Educación Superior. 13-15 de noviembre de 2013, México.

Postman [Online], "Postman | API Development Environment." [Online]. Recuperado de: https://www.getpostman.com/. [Acceso: 05Marzo-2019].

Puma, G. \& Vinicio, M. (2012). Desarrollo del sistema de gestión académica para la Escuela Gonzalo Rubio Orbe de Otavalo (Bachelor's thesis, Sangolquí/ESPE).

Salazar, O., Aguirre, F \& Osorio, J. (2011). Herramientas para el desarrollo rápido de aplicaciones web. Scientia et Technica, 1(47), 254-258.

Salinas, J. (2004). Innovación docente y uso de las TIC en la enseñanza universitaria. International Journal of Educational Technology in Higher Education (ETHE), 1(1).

Sant'Anna, M. (2010). Reportes en .NET con Crystal Reports.

Sierra, F. (2011). Microsoft C\#. Curso de programación (Vol. 7). Grupo Editorial RA-MA.

Tramullas, J. (2006). Software libre para gestión de recursos de información digital. Madrid: Pearson Prentice-Hall.

Trasobares, A. (2003). Los sistemas de información: evolución y desarrollo. Proyecto Social: Revista de Relaciones Laborales, (10), 149-165. 